\title{
A first report of endoscopic ultrasound-guided biopsy in the diagnosis of desmoid-type fibro- matosis
}
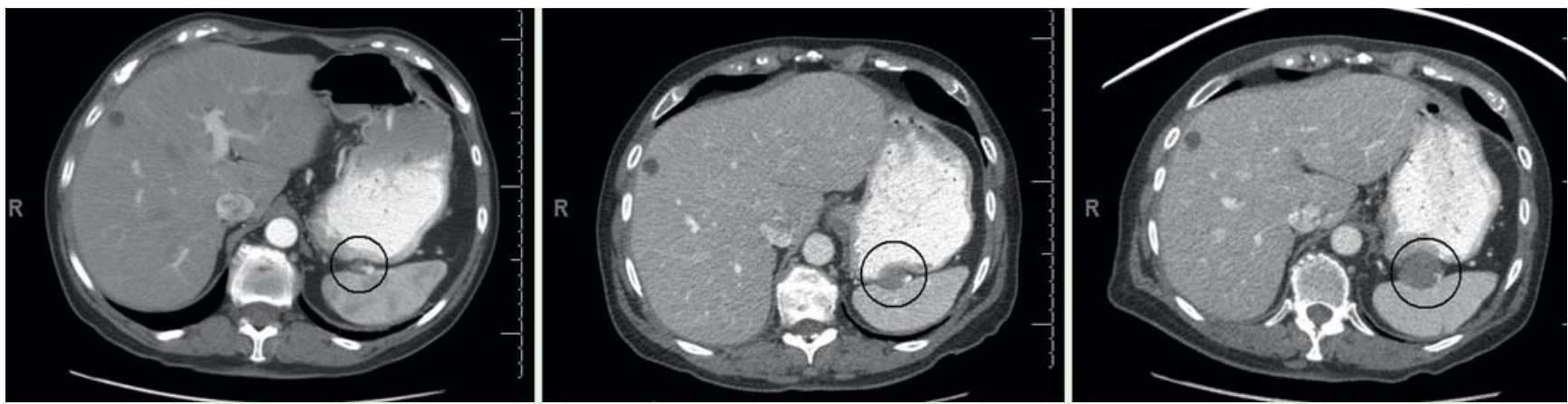

Fig. 1 Serial re-staging abdominal computed tomography (CT) scans showing the gradually increasing size of the splenic hilar soft-tissue mass (circled), which was well-circumscribed and appeared avascular. Also seen is a simple cyst in the liver, which remained stable on these serial scans.

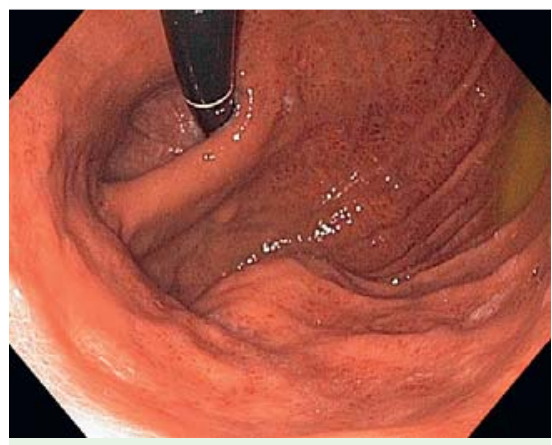

Fig. 2 Esophagogastroduodenoscopy image revealing an approximately $3-\mathrm{cm}$ subepithelial compression in the gastric fundus.

A 65-year-old woman presented with recurrent sporadic mesenteric desmoidtype fibromatosis following a primary margin-positive surgical resection. The disease relapsed with new mesenteric nodules and a splenic hilar mass. Serial computed-tomography (CT) scans in the 4 months after initiation of tamoxifen revealed that the splenic hilar mass was gradually increasing in size ( $\bullet$ Fig. 1 ).

A tissue diagnosis was necessary because radiological interpretation was not entirely consistent with desmoid-type fibromatosis. In the absence of safe access for a CTguided biopsy, the patient was referred for an endoscopic ultrasound (EUS)-guided fine needle aspiration (FNA). Esophagogastroduodenoscopy revealed a fundic subepithelial compression ( $\bullet$ Fig. 2).

EUS revealed a $3.5 \times 2.36-\mathrm{cm}$, hypoechoic, heterogeneous splenic hilar mass with scattered hyperechoic foci ( $\bullet$ Fig. 3).

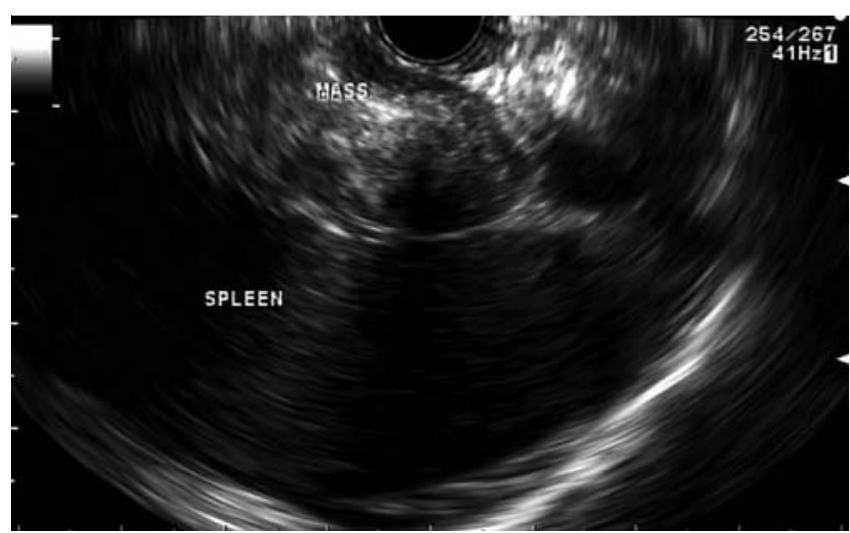

Fig. 3 Endoscopic ultrasound image demonstrating a $3.5 \times 2.36-\mathrm{cm}$, ovalshaped, hypoechoic, heterogeneous splenic hilar mass with well-defined borders and scattered hyperechoic foci.

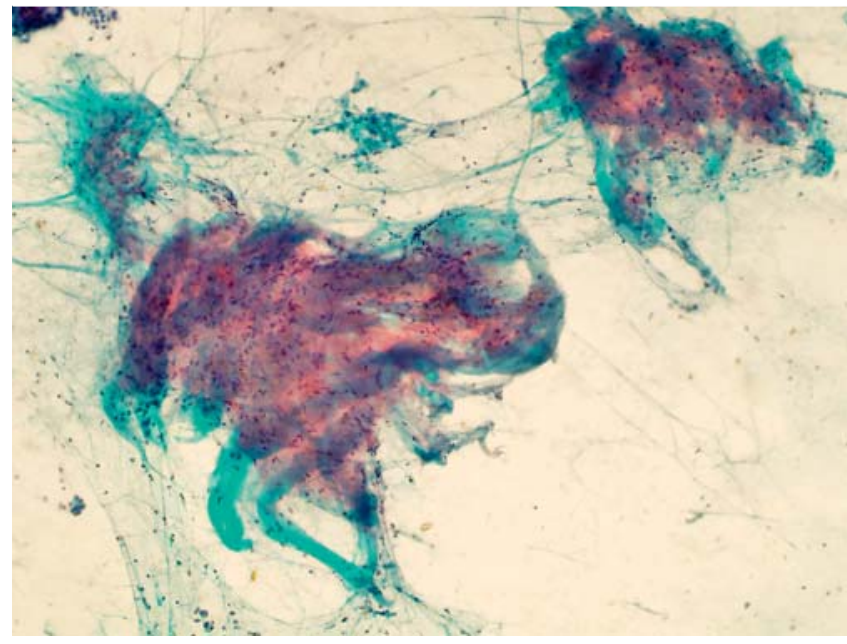

Fig. 4 Cytopathologic appearance using a Papanicolaou stain (magnification $\times 100$ ) of the biopsy specimen taken by endoscopic ultrasound (EUS)-guided fine needle core biopsy (FNCB) showing dense collagenous tissue with a slight increase in the number of bland spindle cells, a morphological appearance that in this patient was compatible with desmoid fibromatosis. Figure courtesy of John Stewart, MD.

Initially attempts were made at FNA with three passes of a 25-gauge needle (EchoTip; Cook Medical Inc., Bloomington, Indiana, USA), all of which were nondiagnostic. Subsequently, an attempt was made at EUS-guided fine needle core biopsy
(FNCB) with two passes of a 22-gauge needle (EchoTip ProCore; Cook Medical). Cytopathology revealed fragments of dense collagenous tissue with bland spindle cells, compatible with desmoid-type fibromatosis ( Fig. 4). 
The patient was considered unresponsive to tamoxifen, therefore imatinib monotherapy was initiated.

Desmoid-type fibromatosis is a rare fibroblastic proliferative disease, which is usually sporadic and very occasionally associated with Gardner's syndrome [1]. Primary surgical resection is the standard treatment, but if resection margins are positive, local recurrence rates are approximately $80 \%$. Treatment options for recurrence include repeat surgery, radiotherapy, tamoxifen, doxorubicin-based regimens, and more recently therapy with imatinib [2,3]. These tumors have a dense fibrous stroma, therefore FNA may not yield diagnostic material [4]. EUS-FNCB with a 19-gauge Quick-Core needle (Cook Medical) can be technically challenging. The new 22-gauge EchoTip ProCore needle features a core-trap and a reverse-bevel for tissue-core procurement [5]. We performed FNCB with the aim of providing better cytology than had been obtained on the FNA specimens. Tissue procurement for histopathology can also be accomplished. To our knowledge, this is the first report of the use of EUS in the imaging and diagnosis of sporadic desmoid-type fibromatosis. Given this experience, we recommend EUS-guided FNCB instead of FNA when sampling suspected lesions with dense fibrous stroma such as desmoid tumors.

Endoscopy_UCTN_Code_CCL_1AF_2AG_3AD

Competing interests: None

\section{S. G. Krishna, R. Suzuki, M. S. Bhutani}

Department of Gastroenterology, Hepatology and Nutrition, University of Texas MD Anderson Cancer Center, Houston, Texas, USA

\section{References}

1 Reitamo JJ, Scheinin TM, Hayry P. The desmoid syndrome. New aspects in the cause, pathogenesis and treatment of the desmoid tumor. Am J Surg 1986; 151: 230 - 237

2 Gega M, Yanagi H, Yoshikawa R et al. Successful chemotherapeutic modality of doxorubicin plus dacarbazine for the treatment of desmoid tumors in association with familial adenomatous polyposis. J Clin Oncol 2006; 24: $102-105$
3 Penel N, Le Cesne A, Bui BN et al. Imatinib for progressive and recurrent aggressive fibromatosis (desmoid tumors): an FNCLCC/ French Sarcoma Group phase II trial with a long-term follow-up. Ann Oncol 2011; 22: $452-457$

4 Owens CL, Sharma R, Ali SZ. Deep fibromatosis (desmoid tumor): cytopathologic characteristics, clinicoradiologic features, and immunohistochemical findings on fine-needle aspiration. Cancer 2007; 111: 166-172

5 Komanduri S, Keswani RN. Feasibility, specimen adequacy, and diagnostic accuracy of a new EUS guided core biopsy needle: a pilot study. Gastrointest Endosc 2011; 73 (Suppl.): AB336

\section{Bibliography}

DOI $10.1055 / \mathrm{s}-0030-1256959$

Endoscopy 2011; 43: E417-E418

(c) Georg Thieme Verlag KG Stuttgart · New York . ISSN 0013-726X

\section{Corresponding author}

\section{S. Bhutani, MD}

Department of Gastroenterology,

Hepatology and Nutrition

University of Texas MD Anderson Cancer Center

1515 Holcombe Blvd.

Houston, Texas 77030-4008

USA

Fax: +1-713-563-4408

Manoop.Bhutani@mdanderson.org 